\title{
Ekkehart Krippendorff
}

\section{Die Idee der Universität}

Gerade weil die - deutsche - Universität gegenwärtig von ihrer materiellen Basis her in Frage gestellt wird, müssen wir von ihrer Idee sprechen. 'Alle reden von Kürzungen - wir nicht' - müßte die trotzige und zugleich selbstbewußte Haltung derer sein, die sich heute noch, oder heute erst recht für die Universität als Lebensform, als Arbeitsplatz und als gesellschaftliches Projekt einsetzen. Fast möchte es scheinen, als handle es sich hier um eine List der Vernunft im Gewande der Unvernunft, daß die rücksichtslosen, blindwütigen Kürzungen der Universitätshaushalte durch eine bornierte, intellektuellenfeindliche politische Klasse und ihre bürokratischen Helfershelfer uns zur Verteidigung und Rechtfertigung dieser Institution nötigen. Verteidigung und Rechtfertigung aber, das heißt zunächst einmal und vor allem: Besinnung - Besinnung darüber, was wir da eigentlich verteidigen und rechtfertigen. Große gesellschaftliche und politische Krisen waren zumindest in Deutschland immer zugleich auch Universitätskrisen, was die besondere Abhängigkeit und Sensibilität dieser Institution von ihrer Umgebung unterstreicht. Nicht immer mußte die Universität »verteidigt« oder »gerechtfertigt« werden - oft war sie auch, und das wäre der Idealfall (ja eigentlich schon vorweggenommen »die Idee der Universität «), der Ort, an dem Gesellschaft und Politik sich verteidigen mußten.

Aus der europäischen Geschichte sind die Universitäten nicht wegzudenken - ja, man wird gute und beste Gründe für die Behauptung beibringen können: Die Universität ist die einzige kulturelle Institution, die hervorgebracht zu haben Europa welt-kulturgeschichtlich stolz sein kann. Alle anderen Institutionen wurden im Zuge der europäischen Welteroberung seit dem 16. Jahrhundert »mit Feuer und Schwert « den unterworfenen Kulturen aufgezwungen - Kirche und Militär, Staat und Eigentumsordnungen, Rechtssysteme und Verwaltungswesen - die Universität allein hatte Vorbildcharakter und wurde sozusagen freiwillig übernommen. Aber davon abgesehen: »Die Idee der Universität« steht am Anfang der frühneuzeitlichen Wiederentdeckung der politischen Freiheit, die aus dem Konflikt zwischen deutschem Kaiser, norditalienischen Kommunen und dem Herrschaftsanspruch des Papstes in Rom hervorging. Es war die Idee der Autonomie, wie sie die Herzogin Matilde (dieselbe, vor deren Burg Canossa 
1077 der deutsche Kaiser Heinrich IV. sich dem Papst unterwerfen mußte) ihren Bologneser Rechtsgelehrten 1088 gewährte zur zeitgemäßen Auslegung des Römischen Rechtes; siebzig Jahre später hat Kaiser Barbarossa dieses Autonomie-Privileg dann noch einmal so unzweideutig bestätigt, daß es nun zum Vorbild auch für alle späteren Gründungen solcher Stätten autonomen, d.h. nicht fremdbestimmten, nicht zweckgebundenen Denkens wurde. Dabei bezog sich das kaiserliche Privileg insbesondere auf die studentische Autonomie, auf deren den Zünften ähnlichen Rechte der Selbstorganisation. Beide Autonomien entstanden und wurden anerkannt als zusammengehörig. Natürlich waren die Gelehrten der frühen Neuzeit nicht »frei« im modernen Sinne bürgerlicher Gesellschaft - sie bewegten sich selbstverständlich im Rahmen und auf dem Boden der katholischen Theologie - aber das war ebenso vereinbar mit der Autonomie der Universität, wie die grundgesetzlich geschützte Lehrfreiheit bekanntlich an die Treue zur »freiheitlich demokratischen Grundordnung « gebunden ist. Und noch eine historische Erinnerung: zwar teilweise hervorgegangen aus dem Studium des Rechts, hatte zumindest die Medizin und bald auch das Studium griechischer (über die Araber vermittelter) Texte eine gleichberechtigte Rolle, weshalb die frühen großen Schulen durchweg studia generalia hieBen und das auch betrieben, mit einem (heute von keinem Erasmus-Programm erreichten) freien Zirkulieren der Gelehrten von einer universitas magistrorum et scholarum - denn so hießen die »Universitäten « zunächst zur anderen. (Der aus dem mittelalterlichen Latein stammende Begriff universitas bezeichnete zunächst nichts anderes als eine beliebige Gemeinschaft oder organisierte Interessengruppe; erst in Verbindung mit den Lehrern und Lernenden erhielt sie ihre heutige Bedeutung, auch wenn schließlich nur die »Universität《 als Name übrigblieb.)

Das also war - stichwortartig - die Geburts-Idee der Universität: Eine selbstbestimmte Gemeinschaft von Lehrern und Schülern, frei von herrschaftlichen Vorgaben oder $»$ Erkenntnisinteressen «, mobil über Landesgrenzen hinweg, eine 'internationale' Kommunität, in der Qualität der Lehre, Reputation der Gelehrten und - immer wieder - das Ausmaß und der Umfang gesicherter Lehr- und Lernautonomie den Ausschlag gab, sich als Lehrender oder Lernender für diese oder jene Stadt zu entscheiden. Die feudalen wie die patrizischen Landesherren und Stadtregierungen aber hatten ihrerseits sowohl ein ökonomisches Interesse daran, Universitäten bei sich zu haben (Studenten brachten Geld), als auch ein »wissenschaftliches«: Gelehrsamkeit konnte zwar bisweilen politisch-ideologisch unbequem sein, langfristig aber war Intelligenz - ausgebildete Juristen, Mediziner, später auch Naturwissenschaftler - von nicht zu unterschätzendem Vorteil für das eigene Land. Gründung und Auflösung, Aufstieg und Niedergang von Universitäten waren, seit dem 15. Jahrhundert, ein einigerma- 
Ben zuverlässiger Index für die gesellschaftliche und geistig-politische Verfassung einer Landesherrschaft oder von Stadtrepubliken. Die große Krise der Reformation und die enormen schöpferischen Energien, die sie zunächst freisetzte, ist ohne ihre Vorbereitung durch die Humanisten an den Universitäten und ohne deren Verarbeitung danach nicht denkbar; die Krise wäre zwar auch so gekommen - aber sie hätte nicht jene konstruktive Entfaltung durchgemacht, die sich in Neugründungen wie Marburg, Königsberg, Jena, später Gießen, Straßburg, Halle, Göttingen u.a.m. manifestierte.

Und eine andere große Krise hat schließlich zur Gründung der »modernen« Universität geführt, an deren Ende wir möglicherweise stehen, die aber gleichwohl zu unserer Geschichte gehört und damit in einer jeden denkbaren Besinnung auf die Idee dieser Institution aufgehoben werden muß, so wie ihre frühneuzeitliche Erbschaft auch: die Friedrich-Wilhelm-Universität in Berlin, die mit dem Namen Wilhelm von Humboldts aufs engste verknüpft ist, aber eben auch mit dem Zusammenbruch und dann der politischen Rekonstruktion, um nicht zu sagen Renaissance Preußens. Diese Universität, 1809 gegründet, wurde vorbildhaft weit über PreußenDeutschland hinaus - und das nicht zuletzt, weil sie in der nun (fast) völligen, jedenfalls aber rechtlich garantierten Lehrfreiheit eines der wichtigsten Erbstücke der 'alten' Universitäts-Idee unter neuen, modernen Bedingungen wiederbelebte, sondern auch weil sie dem die von Klassen- und Standesprivilegien (weitgehend) befreite Lernfreiheit hinzufügte und beides unter der Neuinterpretation der Autonomie als »Einheit von Forschung und Lehre « zusammenfaßte. Es ist nicht mehr nötig, zum x-ten Mal die Idee der Humboldt'schen Universität zu evozieren (und als »überholt« zu kritisieren), diese Diskussion ist ausgiebig genug, so scheint es jedenfalls, geführt worden. Aber wichtig ist es zu erinnern, daß jene Universität ein Kind der Krise war, eine Antwort der großen Gelehrten, der Intellektuellen ihrer Zeit, auf die politisch-moralische Herausforderung und die Erschütterung, die sich in der Französischen Revolution entladen hatte und die ja mit dem napoleonischen Kaiserreich, dessen imperialistischer Eroberungspolitik und deren ruhmlosen Zusammenbruchs ein nicht gerade glückliches Ende genommen hatte. »1789 ff« war eine ebenso große Enttäuschung für die universitas magistrorum et scholarum wie »1989 ff« - aber damals hatte die europäische und nicht zuletzt hier die deutsche Intelligenz die Kraft, oder den Mut, oder die Phantasie, oder das Selbstvertrauen, darauf mit einem konstruktiven Entwurf zu reagieren, denn schließlich war Wilhelm von Humboldt nur einer von vielen illustren Namen, den wir mit dieser 'Rekonstruktion der Politik aus dem Geiste der Universität' verbinden; $\mathrm{da} ß$ das nicht in jeder Hinsicht positiv gesehen werden muß, sei der Vollständigkeit halber angemerkt - die neuen wie die wiederbelebten oder 
transformierten Universitäten wurden nach einigen Jahrzehnten zu Zentren national-patriotischer Agitation und politischer Reaktion, aber sie hatten doch auch ihre, in demokratischer Perspektive großen Augenblicke, wie etwa das Beispiel der »Göttinger Sieben « oder die Notwendigkeit polizeistaatlicher Repression gegen »demokratische Umtriebe« zeigen.

$» 1848 \ll$, die Niederlage der demokratischen Revolutionen überall in Europa, bedeutete für Deutschland zumindest auch die Niederlage der 'Republik der Lehrenden und Lernenden' und die ideologische Unterwerfung der Universität unter den dann kommenden Nationalstaat. Zu ihrem, seiner und unser aller Schaden: denn die wichtigsten Ansätze zu einer kritischen Auseinandersetzung mit dem Industriesystem, mit Kapitalismus, Militarismus und Imperialismus, die Europa dann in die Katastrophe des Ersten Weltkrieges stürzen sollten, die wurden außerhalb der Universität entwikkelt und diskutiert und gelangten darum nie ins Zentrum der Gesellschaft, wurden von den politischen Klassen nur als ihre Privilegien bedrohend wahrgenommen (was auch stimmte), nicht aber als Versuch der Rettung von Humanität und friedlicher Zukunft vor der Entladung der akkumulierten Gewaltpotentiale. Wo die Universität versagte bei der Bearbeitung der großen Themen der Zeit, in dem Maße, in dem sie sich einreihte und anpaßte an die herrschenden Interessen, da verlor die Gesellschaft ihr kritisches Gewissen und ihren wichtigsten Kompaß, die Politik wurde blind. Es hat für die internationale academic community keine beschämendere Zeit gegeben als die des Ersten Weltkrieges, da Studenten und Professoren überall einstimmten in die furchtbaren nationalen Kriegschöre. Und sie hat sich auch danach nur mühsam und mit geringen Erfolgen von dieser Stunde ihrer Unwahrheit erholt.

In Deutschland hat es dann, nach dem Zweiten Weltkrieg bzw. nach der Katastrophe der zwölf Jahre NS-Herrschaft, in die die Universitäten tief verstrickt waren, wenigstens Ansätze gegeben, die Universität neu zu denken. Zwar überwog der Versuch, eine Kontinuität zu konstruieren und die Universität als »im Kern gesund « erscheinen zu lassen, aber es wurde doch indirekt zugegeben, daß die Idee der Universität einer Besinnung bedürfe. Das bestand zum einen darin, daß es fast überall zur Einführung eines »studium generale« kam: nicht-obligatorische aber doch universitäts-gesellschaftliche zentrale Veranstaltungen, die an das humanistische Bildungserbe anzuknüpfen versuchten, also den allgemeinbildenden Charakter der Hochschule thematisierten und einen durchaus vorhandenen entsprechenden Bedarf der Nachkriegs-Studentengeneration befriedigten; besonders aktiv war damals das Land Württemberg mit dem Sozialdemokraten Carlo Schmid als Kultusminister. Eine andere Variante, deren letzte Reste geradezu dieser Tage durch den sog. " Sparzwang « in Berlin eliminiert werden, war die Ergänzung des schmalspurigen, potentiell technokratisch anfälli- 
gen Technikerstudiums an den Technischen Hochschulen durch humanistische Bildungsfächer und die Umwandlung der TH's in Technische Universitäten; dazu gehörte (in Berlin jedenfalls) auch der explizite Verzicht auf jegliche militärdienliche und Rüstungsforschung. - Das waren Ansätze immerhin, und die Idee der Universität wurde jedenfalls über Curricularprobleme hinaus thematisiert und diskutiert.

»1968« darf man, in der Geschichte der Bundesrepublik, ihre »zweite Gründung « nennen, das Nachholen jener demokratischen »Kulturrevolution «, die unter dem Druck der materiellen Nachkriegsprobleme und des Kalten Krieges vergessen worden oder liegengeblieben war. Und da spielten die Universitäten ja nun bekanntermaßen eine zentrale Rolle: Die $\gg$ Studentenrevolte« war vor allem dies: eine 'Liebeserklärung' an die Universität, eine Wiederentdeckung der Universität als zentraler Ort für die Reflexion gesellschaftlicher - politischer, ökonomischer, ethischer - Probleme, eine Rückgewinnung der Universität als Lebensraum, eine Proklamation der Universität als Experimentierfeld schöpferischen Denkens in Freiheit, ein Ernstnehmen der Autonomie, die sich aber zugleich in den Dienst »der Gesellschaft«, sprich: der unterprivilegierten Schichten hier und der ausgebeuteten Menschen in den Ländern der Dritten Welt stellen wollte. Viel ist über $\gg 68$ « geschrieben und geredet worden - nur selten wurde erkannt, daß diese studentische Bewegung in dem, was sie wollte, eine große Niederlage erlitten hat - bei äußerlichen Erfolgen wie der Öffnung der Hochschulen für Arbeiterkinder, zweitem Bildungsweg, der (schrittweise wieder zurückgenommenen) Demokratisierung der inneren Strukturen usw. Der in jahrelangem Kleinkrieg errungene Sieg der konservativen Kräfte über die Reformimpulse war ein Pyrrhussieg: die Universitäten wurden zu einem profillosen Tummelplatz für zu viele Unmotivierte, die mangels anderer beruflicher Optionen die Chance ergriffen, die bequeme Unmündigkeit schulischen Daseins um einige Jahre zu verlängern. Und die »demokratisierte« Gruppe der Hochschullehrer verkümmerte zu einer Gemeinschaft sich selbst reproduzierender Mittelmäßigkeit, von »juste milieu« Professoren, die bestenfalls kluge Langeweile verbreiten und sich von ihren abgesicherten Stellen jeglichen Zorn über bestehende Verhältnisse, der manche, vielleicht sogar viele von ihnen einst motiviert haben mag, haben abkaufen lassen. Querköpfe, welcher politischen Couleur auch immer, bleiben zunehmend draußen und versuchen entweder den Einstieg in die akademische Karriere schon gar nicht mehr (wer wirklich gut ist, findet inzwischen auch außerhalb der verbeamteten Wissenschaft gutbezahlte Positionen), oder werden beizeiten abgeschreckt und verkümmern irgendwo und irgendwie am Rande. Die gegenwärtige deutsche Universität ist weniger von ihren Strukturen her (das auch) krank, als vielmehr von ihrem Personalbestand. Und das ist gravierender, als wenn es sich um institutionelle, durch 
administrative und gesetzgeberische Eingriffe zu korrigierende Malaisen handelte.

Dennoch müssen wir bei diesen ansetzen und auf solche Eingriffe unsere Hoffnung bauen: Wir, oder diejenigen, die die Universität als Ort des Lehrens und des eigenen Lernens nicht willens sind, aufzugeben, müssen uns an den eigenen Haaren aus dem Sumpfe ziehen, das (fast) Unmögliche möglich machen.

Ein Diskurs, eine ernsthafte Debatte ist nötig über die Idee der Universität, die gegenwärtig zu fehlen scheint und deren Fehlen, konfrontiert mit den dramatischen finanziellen Kürzungsauflagen, den meisten Universitätsangehörigen wie ein Luxus, eine Energieverschwendung erscheint; genau das Gegenteil ist der Fall (1809 hätte man nach dieser Logik auch wichtigeres, nämlich die Befreiung von der französischen Besatzung, zu tun gehabt, als die höheren Bildungsanstalten radikal zu reformieren). Denn die Schwäche der Institution Universität in der Konfrontation mit den öffentlichen Finanzen (daß die politische Klasse selbst verantwortlich ist für die Finanzkrise, die sie jetzt als eine Art unvorhersehbarer Naturkatastrophe erscheinen läßt, angesichts derer wir eben alle den 'Gürtel etwas enger schnallen' müssen, steht auf einem anderen Blatt) besteht doch u.a. darin, daß sie selbst sich ihrer Sache nicht mehr sicher ist, sie keine begründete oder begründbare Auskunft mehr geben kann darüber, wer oder was sie ist, warum es sie in dieser - oder in welcher anderen - Form geben muß, welche Aufgaben, welche Funktionen sie für die Gesellschaft erfüllt. Umgekehrt: Die Gesellschaft, »die Politik《 in ihrer seit Jahren kohärenten Borniertheit der Fortschreibung kapitalistisch-technokratischer Entwicklungsperspektiven, weiß was sie - nicht nur von den Universitäten - will: Effizienz, Profitsteigerung, internationale Konkurrenzfähigkeit, technologische Fortschritte, freie Bahn für den individuell Tüchtigen (sprich: den rücksichtslosesten Aufsteiger), den Markt als ausschließliches Erfolgskriterium für Qualität oder gesellschaftliche Relevanz: die Reihe läßt sich logisch leicht fortsetzen.

Die Universität, wenn sie - wie inzwischen weitgehend der Fall - diese Agenda, diese Wertorientierung übernimmt, sich auf sie einläßt, verliert ihre historische und ihre intellektuelle und ethische Identität. Das erneut erforderliche Nachdenken über ihre Geschichte ist kein Luxus. Die Größe und Würde dieser einmaligen »Erfindung « lag - und liegt - in ihrer Autonomie, die von Anfang an mehr und anderes war als nur sozio-politische oder rechtliche Selbständigkeit, vielmehr beinhaltete, keine Dienstleistungsfunktionen zu haben, jedenfalls nicht in einem politisch, ökonomisch oder ideologisch verwertbaren Sinne. Der »Dienst«, den die Universität idealiter (und wir reden ja hier von der 'Idee der Universität') der Gesellschaft und u.U. auch der Politik leistete und leisten kann, besteht darin, ihr einen kritischen Spiegel vorhalten zu können, einen Freiraum darzustellen, 
in dem die Gesellschaft ihre Probleme ohne Entscheidungs- oder Verwertungszwang frei diskutieren kann (oder von dafür freigestellten, qualifizierten Experten diskutieren lassen kann). Und der weitere »Dienst « besteht darin, daß hier junge Leute gebildet werden, die in der Lage sind, Zusammenhänge zwischen augenscheinlich Nicht-Zusammenhängendem herzustellen und dadurch unser Wissen erweitern, ohne unbedingt für jedes »Problem《 gleich eine »Lösung « finden zu müssen. In anderen Worten (und das kann nicht genügend unterstrichen werden): Die Universität hat primär eine generelle Bildungs- und nur sekundär eine Ausbildungsfunktion. Für die Ausbildung von qualifizierten Fachleuten (und wer möchte es leugnen, daß ein Arzt, ein Jurist, ein Brückenbauingenieur nicht eine gute Fachausbildung braucht) sind Fachhochschulen zuständig - und sie bedürfen auch möglicherweise heute der größeren Förderung und des Ausbaus. Aber die Idee der Universität ist eine andere, weniger unmittelbar nützliche, »vermarktbare «, die Idee der Universität ist auch und nicht zuletzt der Wissenschaft verpflichtet.

Die 'Idee der Wissenschaft' gehört darum ganz essentiell in den Diskurs über die Universität. Um diese große Idee in diesem Zusammenhang nicht auf griffige Kurzformeln zu bringen, sei Wissenschaft wenigstens negativ gefaßt: Was heute $\mathrm{zu} \gg 99 \%$ unter diesem Etikett läuft und gefördert wird von Ministerien und Forschungsinstitutionen hat mit Wissenschaft in einem emphatischen, d.h. an Erkenntnis orientierten Sinn in aller Regel nichts zu tun - es ist »Auftragsforschung «, das Ausfüllen von vermeintlichen Wissenslücken, die Lösung eng umschriebener Detailprobleme im Kontext größerer Projekte, deren (ökonomischer) Nutzen nach alles andere als wissenschaftlichen Kriterien festgesetzt und bestimmt worden war. Und es ist darum natürlich kein Zufall, vielmehr sachlogisch verständlich, daß der größte Teil dieser Art wissenschaftlicher Fragen nicht (mehr) von den Universitäten, von Forschungsgruppen in Fachbereichen in der Zusammenarbeit von Lehrenden und Lernenden (die gibt es noch!) bearbeitet und beantwortet wird, sondern von außeruniversitären Instituten, dem Äquivalent $\mathrm{zu}$ den Fachhochschulen der berufsorientierten Ausbildung.

Die Bildungsfunktion der Universität - im deutlichen, wengleich in der Realität des Alltags nicht immer trennbaren Kontrast zur Ausbildungsfunktion - verwirklicht sich in einer weiteren, in den letzten Jahren zunehmend (endlich!) eingeklagten normativen Forderung, nämlich der nach Interdisziplinarität. Was inzwischen als anzustrebendes Ziel, wenn auch beschränkt auf relativ klar umgrenzte Forschungsfragen gilt, ist eigentlich ihr Wesen, die »Idee«, aus der die Universität, die Gemeinschaft der Lehrenden und Lernenden (halten wir erinnernd fest, daß das die Übersetzung der ältesten Selbstdefinition unserer Institution ist: universitas magistrorum et scholarum) hervorging: sie bot studia generalia, allgemeine Studien, das 
Erlernen der wissenschaftlichen Methoden - in der deutschsprachig größten Dramenfigur, Faust, zur Diskussion gestellt. Ohne die Erinnerung an ein »Studium generale« stirbt die Universität - mit dem Nachdenken über seine mögliche Zukunft könnte sie sich retten.

Die Idee der Universität - die autonome Gemeinschaft von Lehrenden und Lernenden, verbunden zu zweckfreiem Dienst an der Gesellschaft, damit diese sich über die Zwecke öffentlichen Handelns immer wieder Rechenschaft ablegen kann und muß - speist sich nicht nur aus ihrer eigenen Geschichte. Sie hat auch ihre Relevanz für soziologische und lebensperspektivische Veränderungen, die die moderne »postindustrielle« Gesellschaft nur allzu deutlich unterscheiden von ihren früheren Erscheinungsformen. Es ist bezeichnend, daß ein beträchtliches $\mathrm{Ma} \beta$ an Universitäts- oder richtiger »Studentenkritik« dahin geht, die angeblich große Zahl von Studierenden zu beklagen, die nur deswegen immatrikuliert seien, weil sie sich vor der drohenden Arbeitslosigkeit fürchten, also diese nur hinausschieben möchten. Darf, ja muß man diese Beobachtung (die teilweise durchaus berechtigt ist) nicht umkehren und sagen: glücklich die »postindustrielle« Gesellschaft, deren Nachwuchs die Chance gegeben wird, sich ohne den Druck schneller Reproduktion, also zielstrebiger Berufsausbildung, an der Universität zweckfrei zu bilden, der Neugier des Wissenwollens um seiner selbst willen nachzugehen, zu studieren aus Freude an der Sache und nicht um eines aufstiegsrelevanten Titels willen? Und das gilt auch in ähnlichem Maße dann für ältere Generationen, die entweder das Berufsleben hinter sich haben, aber gleichwohl noch immer und für ein bis zwei und mehr Jahrzehnte noch aktive Bürger ihrer Städte und Gemeinden sind, oder aber ihr Berufsleben unterbrechend sich den Luxus von einigen Universitätssemestern gönnen können. Die Idee der Universität ist so groß und fruchtbar, daß sie auch und gerade auf neue soziologische Herausforderungen reagieren kann, wenn man sie läßt ...

Nein, nicht »wenn man sie läßt«, sondern wenn die Universität einen Begriff von sich selbst wiedererlangt, sie sich über sich selbst zu verständigen beginnt, einen Prozeß der selbstkritischen Reflexion über ihren historischen, politischen, soziologischen und wissenschaftlichen Standort einleitet, erst dann wird sie mit Selbstbewußtsein als Fordernde, nicht als Bittstellernde in der Öffentlichkeit auftreten können. Der mangelnde gesellschaftliche Rückhalt, der ihre Position im gegenwärtigen Kampf um die Ausgaben der öffentlichen Hand kennzeichnet, geht auch auf ihr eigenes, selbstgemachtes Konto. In den »fetten Jahren « haben sich ihre Angehörigen nicht oder doch viel zu wenig um ihre eigene Institution gekümmert; das letzte Mal, daß das in Deutschland - und nicht nur hier - der Fall war, war in den Jahren um 1968. Damals haben die beamteten Professoren alles getan, diesen kreativen Impuls, der von engagierten Studierenden ausging, $\mathrm{zu}$ blockieren und mit Hilfe der staatlichen Institutionen dann umzufunk- 
tionieren in eine entmündigte Reform; den Rest besorgte der Bildungswohlstand der 70er und 80er Jahre. Trotzdem ist es nicht - ist es nie - zu spät, aus den Fehlern der Vergangenheit so gut wie aus ihren Versprechungen zu lernen und Kraft zu schöpfen. Das Potential der Universität ist besser als ihr Ruf, ihre Idee ist so groß und aufregend, wie sie es über die Jahrhunderte immer gewesen ist. Es kommt nur darauf an, sich ihrer wieder zu besinnen und sie zur Diskussion zu stellen.

\section{PROKLA 105 (Dezember 1996): Staatsbürgerschaft}

Die Staatsbürgerschaft befindet sich am Schnittpunkt von Staat und Individuum, sie verbindet Bürger und politisches Gemeinwesen, wobei die Zugehörigkeit zu diesem Gemeinwesen im herrschenden Diskurs über Nationalität definiert wird. Gerade diese Verbindung von Nationalität und Staatsbürgerschaft wird zu einem politisch zunehmend umkämpften Feld. Während die politische Fragmentierung Osteuropas die Bedeutung der Nationalität wieder verstärkt, wird in der Europäischen Union das Konzept einer Unionsbürgerschaft diskutiert. Unklar ist auch die Auswirkung der allseits konstatierten Globalisierungsprozesse auf das nationalstaatlich beschränkte Konzept der Staatsbürgerschaft. Und schließlich kann auch im Innern von Nationalstaaten keineswegs von einer homogenen nationalen Zugehörigkeit ausgegangen werden, so daß nicht nur die Herstellung von Gleicheit, sondern auch die Anerkennung von Differenz auf der Agenda zivilgesellschaftlicher Forderungen erscheint.

\section{PROKLA 106 (März 1997): Klassen und Politik in Deutschland}

In den gewerkschaftlichen Debatten gelten Linke vielfach als ewiggestrige " Traditionalisten «, diejenigen, die nicht nur auf Kooperation mit dem Kapital, sondern gleich auf Co-Management setzen dagegen als entschiedene »Modernisierer «. Bei den Unternehmern wird nicht nur der Flächentarifvertrag in Frage gestellt, sondern die Existenz der eigenen Verbände gleich mit. Das Verhältnis von Lohnarbeit und Kapital scheint sich in Deutschland neu zu strukturieren. Ist dies alles nur eine Anpassung an die europäisch-nordamerikanische Normalität oder drücken sich hier grundsätzliche Veränderungen aus? In Deutschland nehmen die Geldvermögen nicht nur immer schneller zu, die Politik unterwirft sich auch immer stärker der Logik ihrer Verwertung. Die Kehrseite des privaten Reichtums ist die öffentliche Armut. Welche Rolle kann unter diesen Umständen noch ein Sozialstaat spielen? Leistet sich jetzt auch der entwickelte deutsche Kapitalismus die langfristige Exklusion einer »Underclass «?

Beiträge zu diesem Heft sind gesucht. Exposés (auf Diskette oder per e-mail) sollten bis spätestens 15. 11. 96 bei der Redaktion eingehen, Redaktionsschluß für die fertigen Artikel ist der 5. 1. 1997. 